\title{
AVALIAÇÃO DO ESTADO NUTRICIONAL E RISCO NUTRICIONAL EM IDOSAS DO PROGRAMA UCS SENIOR
}

\author{
Priscila Carra ${ }^{1}$ \\ Josiane Siviero ${ }^{2}$
}

resumo

A avaliação do estado nutricional e a detecção precoce dos riscos nutricionais que acompanham o envelhecimento feminino são consideradas prioritárias na atenção primária à saúde. Este estudo teve como objetivo avaliar o estado nutricional e o risco nutricional em uma amostra de idosas participantes do Programa UCS Sênior da Universidade de Caxias do Sul. Trata-se de um estudo transversal, descritivo e analítico. Utilizaram-se variáveis sociodemográficas, antropométricas, epidemiológicas, consumo alimentar obtido através do questionário de frequência alimentar (QFA) e a triagem de risco nutricional (NSI). Dentre as 104 avaliadas, 59,6\% possuíam companheiros, 71,1\%

1 Nutricionista graduada pela Universidade de Caxias do Sul (UCS-RS). Pós-graduada em Nutrição Esportiva e Fisiologia do Exercício pelo Centro Universitário da Serra Gaúcha (FSG). E-mail: pry_carra@hotmail.com.

2 Nutricionista graduada pela Universidade Vale do Rio dos Sinos. Mestre em Gerontologia Biomédica pelo Instituto de Geriatria e Gerontologia (IGG-PUCRS). Doutora em Ciências da Saúde - ênfase em Geriatria pela Faculdade de Medicina da PUCRS. Docente da Universidade de Caxias do Sul (UCS-RS). E-mail: josianesiviero@hotmail.com. 
referiram possuir ensino fundamental completo, médio ou superior, 74\% possuíam renda mensal acima de dois salários mínimos e 94,2\% classificaram-se como ativas e/ou independentes. Em relação ao IMC, 44,2\% estavam com pré-obesidade e/ou obesidade e apenas $11,5 \%$ baixo peso. Houve prevalência elevada de dislipidemias (61,5\%) e circunferência da cintura em risco para doenças cardiovasculares (74\%), segundo classificação da OMS (2003). Dentre as variáveis estudadas, somente a depressão aumentou significativamente com o aumento do risco nutricional pela classificação do NSI ( $p=0,002)$. O Nutrition Screening Initiative identificou 51,9\% de idosas em baixo risco, 36,5\% risco moderado e 11,5\% alto risco nutricional. Identificar fatores preditivos ao risco nutricional, através da associação de indicadores, pode permitir o direcionamento de intervenções de saúde, especialmente a nutricional, além de contribuir com a qualidade de vida dessa crescente parcela populacional.

palavras-chave

Envelhecimento. Atenção Primária à Saúde. Estado Nutricional. Risco.

\section{Introdução}

Nas últimas décadas, as transformações sociais e econômicas e suas consequentes alterações nos estilos de vida, mudança nos hábitos alimentares e, ainda uma maior expectativa de vida da população mundial, implicam no que diz respeito à prestação de cuidados de saúde adequados e à necessidade latente de prevenir os principais contribuintes para o risco nutricional (ATELLA; CONTI, 2014). Dessa forma, o processo do envelhecimento é caracterizado por alterações fisiológicas, metabólicas, nutricionais e psicossociais que influenciam nas condições de saúde e no estado nutricional dos idosos (GONÇALVES et al., 2012).

Muitos são os fatores de risco que ameaçam a população idosa, dentre os quais, estão a presença de doenças crônicas e suas comorbidades (TANJANI et al., 2015), consequentemente associadas ao consumo de medicamentos que podem causar complicações adversas (MARENGONI et al., 2014). Alterações psicossociais como isolamento social, depressão (HEUBERGER; WONG, 2014), tabagismo e/ou etilismo (KUERBIS et al., 2014), comprometimento cognitivo, fisiológico (ZOGHBI et al., 2013; BRETON et al., 2014) e dificuldades econômicas também podem influenciar na desmotivação do idoso e impor restrições sobre a preparação e o consumo de alimentos saudáveis (DEAN et al., 2009). Nesse cenário, uma variedade de ferramentas de triagem nutricional foram 
desenvolvidas e amplamente recomendadas em diferentes ambientes de cuidado, na tentativa de identificar e avaliar precocemente os idosos em situação de risco ou déficit da ingestão alimentar, a fim de evitar principalmente o aparecimento ou até mesmo auxiliar no tratamento de doenças desse segmento populacional (YAP; NITI, 2007).

Com o objetivo de investigar prováveis alterações nutricionais de indivíduos com idade superior a 65 anos, foi publicado em 1991, nos Estados Unidos da América (EUA), o Nutrition Screening Initiative - NSI (Iniciativa de Triagem Nutricional). Trata-se de uma avaliação subjetiva, composta por dez questões de sinais de alerta, que pode ser aplicada ou respondida pelo idoso, familiar ou cuidador. O resultado da pontuação permite diferenciar de maneira relativamente acurada, rápida e pouco onerosa, o risco do estado nutricional (POSNER et al., 1993). Embora essa ferramenta não tenha sido destinada a substituir as avaliações nutricionais existentes e possua eficiência limitada quanto à predição de risco de mortalidade, é considerada uma importante estratégia de saúde pública na atenção primária (SANTOS et al., 2013). Sendo assim, esse estudo teve como objetivo avaliar o estado nutricional e o risco nutricional através do questionário (NSI) em idosas participantes do Programa UCS Sênior da Universidade da Terceira Idade da Universidade de Caxias do Sul.

\section{Métodos}

Foi realizado um estudo do tipo transversal, descritivo e analítico. Os dados foram coletados no período de 2010 a 2014. A amostra foi composta por 104 mulheres, com idade maior ou igual a sessenta anos, participantes do Projeto Nutrição e Envelhecimento (NUTENV), intitulado "Alimentação e Envelhecimento: estudo do perfil nutricional, antropométrico e a relação com fatores socioeconômicos e culturais de idosos participantes da Universidade da Terceira Idade - UNTI", aprovado pelo Comitê de Ética e Pesquisa da Universidade de Caxias do Sul (UCS), sob o número 061/2009. Trata-se de um programa institucional extensionista que presta atendimento qualificado às pessoas com cinquenta anos ou mais, com foco em educação e longevidade.

Tendo base em um questionário estruturado, nutricionistas e/ou estudantes de nutrição previamente treinados, obtiveram informações que deram origem ao banco de dados, por meio de entrevista individual e mensuração antropométrica no Laboratório de Educação e Avaliação Nutricional da UCS, coletadas em aproximadamente trinta a sessenta minutos. Todas as participantes assinaram um Termo de Consentimento Livre e Esclarecido. 
Os dados de identificação e sociodemográficos selecionados foram: faixa etária, estado civil, escolaridade, arranjo familiar, moradia e renda per capita mensal em salários mínimos (s.m). Dentre as variáveis antropométricas utilizou-se peso $(\mathrm{kg})$ e altura $(\mathrm{m})$ com a finalidade de calcular o índice de massa corporal $\left(\mathrm{IMC}=\mathrm{kg} / \mathrm{m}^{2}\right.$ ), além da medida da circunferência da cintura $(\mathrm{CC}=$ $\mathrm{cm})$. O peso corporal foi aferido por balança mecânica da marca Cauduro ${ }^{\circ}$, previamente calibrada. A estatura foi obtida através do estadiômetro acoplado à mesma balança. Os indivíduos foram pesados e medidos de acordo com os procedimentos adotados pelo Sistema de Vigilância Alimentar e Nutricional (SISVAN, 2011). O IMC para idosas foi classificado de acordo com os pontos de corte preconizados pela Organização Pan-Americana da Saúde (OPAS, 2001) e a CC foi classificada segundo a Organização Mundial de Saúde (OMS, 2003).

Em relação às condições de saúde das avaliadas, obteve-se o relato da existência de patologias atuais diagnosticadas pelo médico. Quanto ao nível de capacidade funcional, adaptou-se segundo Matsudo (2000) em atleta, ativa/ independente e frágil/sem dependência.

Por meio de triagem nutricional realizada através do Nutrition Screening Initiative - NSI, investigou-se a presença de doenças e problemas de saúde bucal que dificultavam a alimentação, falta de apetite, consumo de frutas, verduras e/ou laticínios, ingestão de bebidas alcoólicas, condições econômicas, contato social, consumo de medicamentos, alterações no peso e incapacidade funcional. Para cada pergunta foi atribuído uma pontuação que variou de 1 a 4, e a partir do somatório de pontos as idosas foram classificadas em escores: (0-2) baixo risco, (3-5) moderado risco e ( $\geq 6$ ) alto risco nutricional (SANTOS et al., 2013). Através do questionário de frequência alimentar (QFA), obteve-se dados sobre o consumo (uma a três vezes/semana, de três a seis vezes/semana, diariamente ou não consome) de frutas, vegetais crus e cozidos, além de produtos lácteos (leite, queijo e iogurte).

Após a seleção dos dados, realizou-se análises no programa SPSS® (Statistical Package for the Social Science) versão 21.0 e o nível de significância adotado foi $5 \%(\mathrm{p} \leq 0,05)$. Posteriormente, realizaram-se testes estatísticos em que as variáveis quantitativas foram descritas por média e desvio padrão e as variáveis categóricas foram descritas por frequências absolutas e relativas. Para comparar médias entre os grupos, a Análise de Variância (ANOVA) de uma via foi aplicada. Na comparação de proporções, os testes Qui-Quadrado de Pearson ou exato de Fischer foram utilizados. 
Dentre as 104 idosas participantes da pesquisa, conforme Tabela 1, observou-se que $71,2 \%$ possuíam faixa etária entre $60-69$ anos e a idade média foi $67,6 \pm 6,1$ anos. Houve uma tendência de aumento de risco nutricional em mulheres nessa faixa etária $(\mathrm{p}=0,089)$, porém não foi significativa.

Em relação ao estado civil, verificou-se que $59,6 \%$ possuíam companheiro, sendo que $75 \%$ das idosas moravam com cônjuge e/ou familiares ou outros. $\mathrm{Na}$ análise comparativa do item 7 do NSI, observou-se associação significativa com o estado civil e arranjo familiar $(\mathrm{p}<0,001)$. As idosas sem companheiro e que moravam sozinhas, afirmaram realizar na maioria das vezes as refeições sozinhas (figuras 1a e 1b).

Referente à renda, $74 \%$ das idosas possuíam renda mensal maior que $2 \mathrm{s.m}$ e a maioria $(97,1 \%)$ possuía moradia própria. Quanto ao nível de escolaridade, $71,1 \%$ referiram possuir ensino fundamental completo, médio ou superior.

As variáveis antropométricas encontram-se na Tabela 1 . Ao analisarmos o IMC, a média foi $28,3 \pm 4,9$. De acordo com a classificação da OPAS para o IMC, $44,2 \%$ das participantes estavam com pré-obesidade e/ou obesidade e apenas $11,5 \%$ com baixo peso. Quanto à CC, $74 \%$ classificaram-se em risco elevado e/ou muito elevado para doenças cardiovasculares.

Entre as patologias relatadas, as que apresentaram maior frequência foram a dislipidemia com $61,5 \%$ e hipertensão com $58,7 \%$. Conforme Tabela 1 , verificou-se associação estatisticamente significativa do risco nutricional do NSI com depressão $(\mathrm{p}=0,002)$, no qual a prevalência de depressão aumenta significativamente com o aumento do risco nutricional.

Em se tratando do nível funcional, a maioria delas $(94,2 \%)$ classificaram-se como ativas/independentes, 3,7\% frágeis/sem dependência e 1,9\% atleta (Tabela 1). Através do item 10 do NSI que investigou a existência de algum problema de saúde (físico) que incapacitasse de comprar comida, não se obteve associação significativa com o nível funcional $(\mathrm{p}=0,970)$. Além disso, por meio do NSI, $51,9 \%$ das idosas apresentaram baixo risco, $36,5 \%$ moderado risco e $11,5 \%$ alto risco nutricional. 
Tabela 1 - Classificação de risco nutricional do NS/ de acordo com as variáveis sociodemográficas, antropométricas e epidemiológicas.

\begin{tabular}{|c|c|c|c|c|c|}
\hline Variáveis* & $n=104$ & $\begin{array}{c}\text { Baixo risco } \\
\mathrm{n}=54(51,9 \%) \\
\mathrm{n}(\%)\end{array}$ & $\begin{array}{c}\text { Moderado risco } \\
\mathrm{n}=38(36,5 \%) \\
\mathrm{n}(\%)\end{array}$ & $\begin{array}{c}\text { Alto risco } \\
\mathrm{n}=12(11,5 \%) \\
\mathrm{n}(\%)\end{array}$ & $\mathrm{p}^{\star \star}$ \\
\hline $\begin{array}{l}\text { Idade (anos) } \\
\text { Faixa etária }\end{array}$ & $67,6 \pm 6,1$ & & & & 0,089 \\
\hline 60 - 69 anos & $74(71,2)$ & $43(79,6)$ & $21(55,3)$ & $10(83,3)$ & \\
\hline $70-79$ anos & $24(23,1)$ & $8(14,8)$ & $14(36,8)$ & $2(16,7)$ & \\
\hline$\geq 80$ anos & $6(5,8)$ & $3(5,6)$ & $3(7,9)$ & $0(0,0)$ & \\
\hline $\begin{array}{l}\text { Estado civil } \\
\text { (com companheiro) }\end{array}$ & $62(59,6)$ & $32(59,3)$ & $24(63,2)$ & $6(50,0)$ & 0,718 \\
\hline Escolaridade & & & & & 0,271 \\
\hline $\begin{array}{l}\text { Sem estudo e/ou ens. } \\
\text { fund. incompleto }\end{array}$ & $30(28,8)$ & $17(31,5)$ & $9(23,7)$ & $4(33,3)$ & \\
\hline $\begin{array}{l}\text { Ensino fundamental } \\
\text { completo }\end{array}$ & $15(14,4)$ & $9(16,7)$ & $6(15,8)$ & $\mathrm{O}(0,0)$ & \\
\hline Ensino médio & $28(26,9)$ & $14(25,9)$ & $8(21,1)$ & $6(50,0)$ & \\
\hline Ensino superior & $31(29,8)$ & $14(25,9)$ & $15(39,5)$ & $2(16,7)$ & \\
\hline Renda (s.m.) & & & & & 0,712 \\
\hline Sem salário e/ou até 1 & $11(10,6)$ & $7(13,0)$ & $3(7,9)$ & $1(8,3)$ & \\
\hline$>1-2$ & $16(15,4)$ & $6(11,1)$ & $8(21,1)$ & $2(16,7)$ & \\
\hline$>2$ & $77(74,0)$ & $41(75,9)$ & $27(71,1)$ & $9(75,0)$ & \\
\hline Arranjo familiar & & & & & 0,532 \\
\hline Cônjuge e/ou familiares & $74(71,2)$ & $40(71,4)$ & $26(68,4)$ & $8(66,7)$ & \\
\hline Sozinha & $26(25,0)$ & $13(24,1)$ & $9(23,7)$ & $4(33,3)$ & \\
\hline Outros & $4(3,8)$ & $1(1,9)$ & $3(7,9)$ & $0(0,0)$ & \\
\hline Moradia (Própria) & $101(97,1)$ & $54(100)$ & $36(94,7)$ & $11(91,7)$ & 0,162 \\
\hline Peso (kg) & $69,2 \pm 11,8$ & $68,3 \pm 10,6$ & $70,7 \pm 12,5$ & $68,3 \pm 15,1$ & 0,613 \\
\hline Altura (m) & $1,57 \pm 0,06$ & $1,58 \pm 0,06$ & $1,55 \pm 0,06$ & $1,54 \pm 0,06$ & 0,010 \\
\hline $\mathrm{IMC}\left(\mathrm{kg} / \mathrm{m}^{2}\right)$ & $28,3 \pm 4,9$ & $27,3 \pm 4,2$ & $29,4 \pm 5,2$ & $29,0 \pm 6,0$ & 0,124 \\
\hline
\end{tabular}




\begin{tabular}{|c|c|c|c|c|c|}
\hline Variáveis* & $n=104$ & $\begin{array}{c}\text { Baixo risco } \\
\mathrm{n}=54(51,9 \%) \\
\mathrm{n}(\%)\end{array}$ & $\begin{array}{c}\text { Moderado risco } \\
\mathrm{n}=38(36,5 \%) \\
\mathrm{n}(\%)\end{array}$ & $\begin{array}{c}\text { Alto risco } \\
n=12(11,5 \%) \\
n(\%)\end{array}$ & $\mathrm{p}^{\star \star}$ \\
\hline $\begin{array}{l}\text { Classificação } \\
\text { IMC - OPAS }\end{array}$ & & & & & 0,399 \\
\hline Baixo peso $(<23$ kg/m²) & $12(11,5)$ & $7(13,0)$ & $4(10,5)$ & $1(8,3)$ & \\
\hline $\begin{array}{l}\text { Peso normal } \\
\left(23-28 \mathrm{~kg} / \mathrm{m}^{2}\right)\end{array}$ & $46(44,2)$ & $29(53,7)$ & $12(31,6)$ & $5(41,7)$ & \\
\hline $\begin{array}{l}\text { Pré-obesidade } \\
\left(>28-30 \mathrm{~kg} / \mathrm{m}^{2}\right)\end{array}$ & $18(17,3)$ & $8(14,8)$ & $8(21,1)$ & $2(16,7)$ & \\
\hline Obesidade (> 30 kg/m²) & $28(26,9)$ & $10(18,5)$ & $14(36,8)$ & $4(33,3)$ & \\
\hline $\mathrm{CC}(\mathrm{cm})-\mathrm{OMS}$ & $87,6 \pm 11,4$ & & & & 0,132 \\
\hline Normal $(<80)$ & $27(26,0)$ & $19(35,2)$ & $7(18,4)$ & $1(8,3)$ & \\
\hline Elevada $(80-87,9)$ & $33(31,7)$ & $16(29,6)$ & $11(28,9)$ & $6(50,0)$ & \\
\hline Muito elevada ( $\geq 88$ ) & $44(42,3)$ & $19(35,2)$ & $20(52,6)$ & $5(41,7)$ & \\
\hline \multicolumn{6}{|l|}{ Patologias } \\
\hline Diabetes Mellitus & $13(12,5)$ & $4(7,4)$ & $6(15,8)$ & $3(25,0)$ & 0,185 \\
\hline Hipertensão & $61(58,7)$ & $29(53,7)$ & $23(60,5)$ & $9(75,0)$ & 0,382 \\
\hline Osteoporose & $35(33,7)$ & $16(29,6)$ & $13(34,2)$ & $6(50,0)$ & 0,400 \\
\hline Dislipidemia & $64(61,5)$ & $37(68,5)$ & $21(55,3)$ & $6(50,0)$ & 0,298 \\
\hline Depressão & $35(33,7)$ & $10(18,5)$ & $18(47,4)$ & $7(58,3)$ & $0,002^{* \star}$ \\
\hline Câncer & $9(8,7)$ & $5(9,3)$ & $3(7,9)$ & $1(8,3)$ & 0,973 \\
\hline Nível funcional & & & & & 0,653 \\
\hline Atleta & $1(1,0)$ & $1(1,9)$ & $0(0,0)$ & $0(0,0)$ & \\
\hline Ativo/independente & $98(94,2)$ & $51(94,4)$ & $35(92,1)$ & $12(100)$ & \\
\hline Frágil/sem dependência & $5(4,8)$ & $2(3,7)$ & $3(7,9)$ & $0(0,0)$ & \\
\hline
\end{tabular}

Legenda: $n$ = número; \% = por cento; ens. = ensino; fund. = fundamental; s.m. = salário mínimo; $\mathrm{Kg}$ = quilograma; $\mathrm{m}$ = metro; $\mathrm{m}^{2}$ = metro quadrado; IMC = índice de massa corporal;

OPAS = Organização Pan-Americana da Saúde; CC = circunferência da cintura; cm = centímetro; OMS = Organização Mundial de Saúde; NSI = Nutrition Screening Initiative; *Contínuas descritas por média \pm desvio padrão e qualitativa por $n(\%) ;{ }^{* *}$ associação estatisticamente significativa $(p \leq 0,05)$.

Fonte: Tabela elaborada pelas autoras.

Na Tabela 2, observou-se associação significativa de sete itens do NSI com a classificação do escore (baixo, moderado e alto risco), com exceção das questões quatro, seis e sete. Para as outras questões, à medida que aumentava o risco nutricional, aumentava o percentual de respostas positivas. 
Tabela 2 - Distribuição das assertivas dos itens do questionário NS/ segundo o risco nutricional.

\begin{tabular}{|c|c|c|c|c|c|}
\hline $\begin{array}{l}\text { Questões NSI } \\
\text { Pontuação atribuída* }\end{array}$ & $\begin{array}{c}\text { Amostra Total } \\
(\mathrm{n}=104) \\
\mathrm{n}(\%)\end{array}$ & \begin{tabular}{|} 
Baixo Risco \\
$(\mathrm{n}=54)$ \\
$\mathrm{n}(\%)$
\end{tabular} & $\begin{array}{c}\text { Moderado Risco } \\
(\mathrm{n}=38) \\
\mathrm{n}(\%)\end{array}$ & $\begin{array}{c}\text { Alto Risco } \\
(\mathrm{n}=12) \\
\mathrm{n}(\%)\end{array}$ & $\mathrm{p}^{\star \star}$ \\
\hline $\begin{array}{l}\text { 1- Tem alguma doença que } \\
\text { dificulte a sua alimentação } \\
\text { ( } 2 \text { pontos) }\end{array}$ & $18(17,3)$ & $1(1,9)$ & $12(31,6)$ & $5(41,7)$ & $<0,001^{\star \star}$ \\
\hline $\begin{array}{l}\text { 2- Tem comido menos } \\
\text { ultimamente, por falta de } \\
\text { apetite ( } 3 \text { pontos) }\end{array}$ & $13(12,5)$ & $0(0,0)$ & $9(23,7)$ & $4(33,3)$ & $<0,001^{\star \star}$ \\
\hline $\begin{array}{l}\text { 3- Come poucas frutas, } \\
\text { verduras e/ou produtos } \\
\text { derivados do leite } \\
\text { (2 pontos) }\end{array}$ & $13(12,5)$ & $4(7,4)$ & $4(10,5)$ & $5(41,7)$ & $0,005^{\star \star}$ \\
\hline $\begin{array}{l}\text { 4- Ingere mais de } 3 \text { copos } \\
\text { de bebidas alcoólicas/dia } \\
\text { ( } 2 \text { pontos) }\end{array}$ & $\mathrm{O}(0,0)$ & $\mathrm{O}(0,0)$ & $\mathrm{O}(0,0)$ & $\mathrm{O}(0,0)$ & - \\
\hline $\begin{array}{l}\text { 5- Tem problemas na boca } \\
\text { ou nos dentes que dificulte } \\
\text { a alimentação ( } 2 \text { pontos) }\end{array}$ & $15(14,4)$ & $2(3,7)$ & $8(21,1)$ & $5(41,7)$ & $0,001^{\star \star}$ \\
\hline $\begin{array}{l}\text { 6- Tem condições } \\
\text { financeiras para comprar } \\
\text { comida (4 pontos) }\end{array}$ & $104(100)$ & $54(100)$ & $38(100)$ & $12(100)$ & - \\
\hline $\begin{array}{l}\text { 7- Faz as refeições sozinho } \\
\text { na maior parte das vezes } \\
\text { ( } 1 \text { ponto) }\end{array}$ & $41(39,4)$ & $18(33,3)$ & $15(39,5)$ & $8(66,7)$ & 0,102 \\
\hline $\begin{array}{l}\text { 8- Ingere } 3 \text { ou mais } \\
\text { remédios sob prescrição } \\
\text { médica/dia ( } 1 \text { ponto) }\end{array}$ & $64(61,5)$ & $25(46,3)$ & $28(73,7)$ & $11(91,7)$ & $0,002^{* *}$ \\
\hline $\begin{array}{l}\text { 9- Emagreceu ou } \\
\text { engordou pelo menos } 5 \mathrm{Kg} \\
\text { nos últimos } 6 \text { meses } \\
\text { ( } 2 \text { pontos) }\end{array}$ & $22(21,2)$ & $2(3,7)$ & $14(36,8)$ & $6(50,0)$ & $<0,001^{\star \star}$ \\
\hline $\begin{array}{l}\text { 10- Possui algum } \\
\text { problema de saúde (físico) } \\
\text { que incapacite de comprar } \\
\text { comida (2 pontos) }\end{array}$ & $1(1,0)$ & $0(0,0)$ & $\mathrm{O}(0,0)$ & $1(8,3)$ & $0,021^{\star \star}$ \\
\hline
\end{tabular}

Legenda: NSI = Nutrition Screening Initiative; *Para cada questão do NSI é atribuída uma pontuação que varia de 1 a 4. O NS/ categoriza pontuações de 0-2 como baixo risco, 3-5 moderado risco e $\geq 6$ alto risco; $\mathrm{n}$ = número; \% = por cento; $\mathrm{Kg}$ = quilograma, **associação estatisticamente significativa $(\mathrm{p} \leq 0,05)$.

Fonte: Tabela elaborada pelas autoras. 
Os resultados do consumo alimentar obtidos através do (QFA) demonstraram que a maioria das mulheres consumiam todos os dias alimentos como frutas, vegetais crus, leite e queijo. Para os demais alimentos selecionados, iogurte e vegetais cozidos, o consumo foi menor, sendo uma a três vezes por semana. Houve associação significativa entre o consumo alimentar de frutas com o item 3 do NSI $(\mathrm{p}=0,016)$. As idosas que afirmaram consumir poucas frutas, verduras e/ou produtos derivados do leite eram as que menos frequentemente consumiam frutas pelo QFA. A frequência do consumo de verduras e/ou derivados do leite não se associou com o item 3 do NSI (figura 1c).

Figura 1 - Associação do estado civil, arranjo familiar e consumo alimentar de frutas com o NSI.

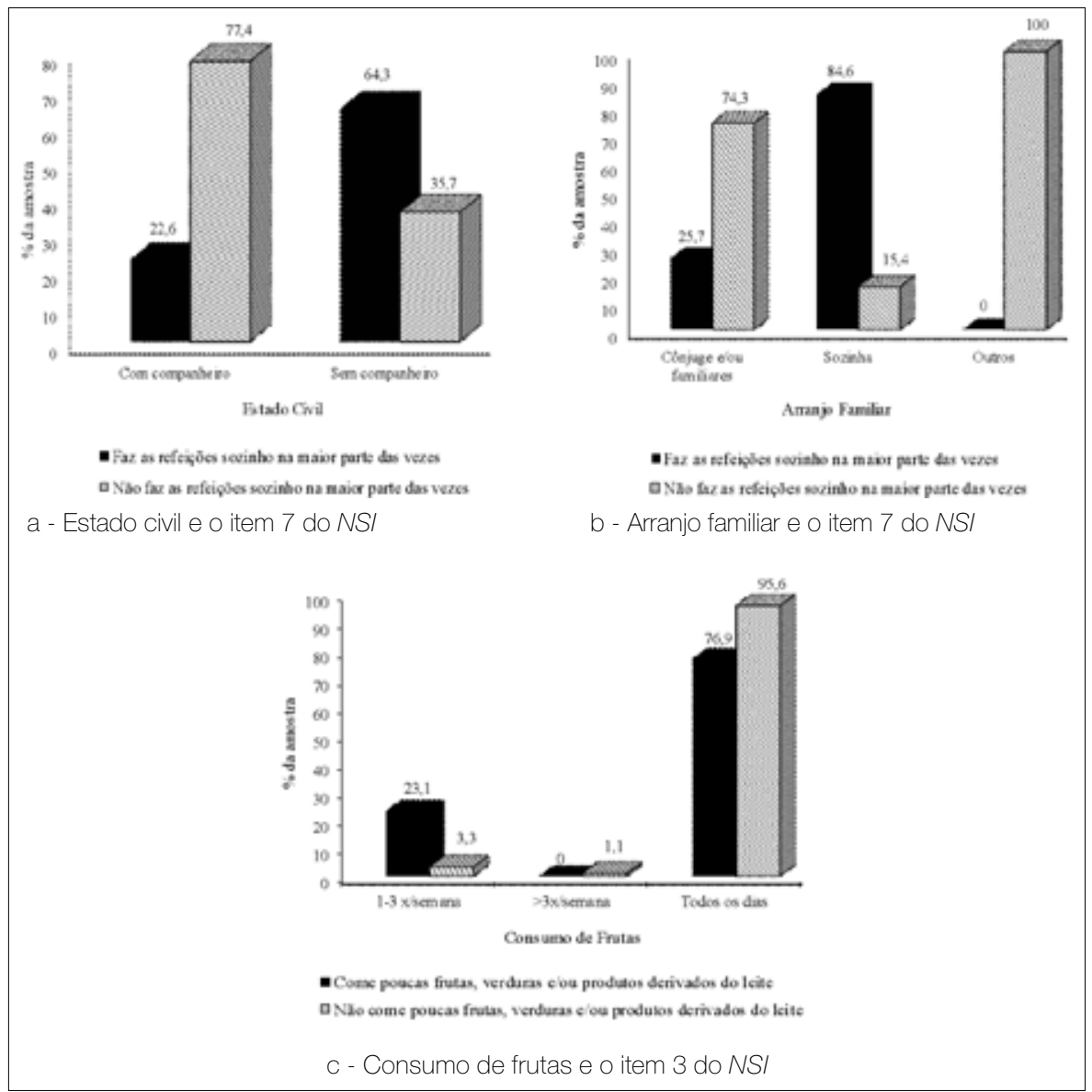

Fonte: Figura elaborada pelas autoras. 
A feminização mundial do envelhecimento e o número de mulheres viúvas, com mais de setenta anos está aumentando progressivamente em relação a outras faixas etárias (DIGIACOMO et al., 2015). Ao contrário do presente estudo, a maioria das idosas avaliadas $(71,2 \%)$ pertenciam à faixa etária dos 60-69 anos, possuíam companheiro e não moravam sozinhas.

$\mathrm{Na}$ faixa etária entre 60-69 anos, observou-se que as mulheres apresentaram uma tendência de aumento de risco nutricional pelo NSI. Os resultados diferem do estudo de Sherina et al. (2004), no qual identificaram em idosos com idade maior ou igual a setenta anos maior prevalência de risco nutricional moderado e alto.

Dentre as idosas com companheiro, $77,4 \%$ afirmavam não realizar as refeições sozinhas. As idosas sem companheiro, assim como as que moravam sozinhas geralmente faziam as refeições de forma isolada. Wham et al. (2014), em Nova Zelândia, identificou aumento significativo de risco nutricional em idosas sem companheiro e que viviam sozinhas. Esse resultado pode estar associado ao fato de que morar com alguém aumenta a oportunidade de compartilhar as refeições e, consequentemente, a variedade do consumo alimentar.

Os resultados do perfil de renda da maioria das participantes eram maiores que dois salários mínimos, sendo que a maioria possuía habitação própria. Esses fatores apontam que as avaliadas possuíam condições de investir na sua qualidade de vida e saúde, na compra dos gêneros alimentícios, podendo, inclusive ser reflexo da alta escolaridade do grupo investigado. Corroborando com esse estudo, Wunderlich et al. (2012) observaram que maiores níveis educacionais e econômicos têm sido associados a melhores condições nutricionais e de saúde em idosos. Segundo DiGiacomo et al. (2015), apesar dos avanços, ainda se constata em nível mundial, um baixo nível socioeconômico e educacional para a maioria das idosas.

Em relação à média de $\mathrm{IMC}$ encontrado $\left(28,3 \pm 4,9 \mathrm{~kg} / \mathrm{m}^{2}\right)$, a maioria das mulheres ficou em pré-obesidade e apresentou risco elevado para doenças cardiovasculares, com CC média de 87,6 $\pm 11,4 \mathrm{~cm}$. Esses valores ficaram parecidos quando comparados com o estudo de Pereira et al. (2009) realizado na Universidade Federal de Santa Maria, onde idosas ativas apresentaram IMC médio de $28,40 \pm 0,82 \mathrm{~kg} / \mathrm{m}^{2}$ e CC média de $92,50 \pm 1,25 \mathrm{~cm}$. Pesquisa de Kim et al. (2011) também identificou em idosos coreanos, sobretudo em mulheres, valores de CC acima do limite recomendado pela OMS $(86,4 \pm 9,0 \mathrm{~cm})$.

Os achados desse estudo demonstraram semelhantes proporções de idosas classificadas com peso normal $(44,2 \%)$ e pré-obesidade/obesidade $(44,2 \%)$ 
de acordo com a OPAS, restando apenas 11,5\% com baixo peso. Em pesquisa realizada em Santos-SP, as idosas avaliadas foram classificadas de maneira semelhante, apenas $37 \%$ apresentaram peso adequado, $49,1 \%$ pré-obesidade/ obesidade e 13,9\% baixo peso (AZEVEDO, D.; AZEVEDO, C., 2014). Esses dados podem refletir no fenômeno de transição nutricional, com repercussões clínicas, biológicas e sociais importantes, principalmente entre os idosos (ANDRADE et al., 2012).

Na análise do perfil de morbidades, destaca-se a presença frequente de doenças crônicas entre as avaliadas, principalmente as dislipidemias (61,5\%), seguida de hipertensão (58,7\%). Ocorreu menor prevalência de depressão e osteoporose, sendo em ambas 33,7\%, diabetes mellitus (12,5\%) e câncer (8,7\%). No estudo de Stobbe et al. (2005), verificou-se um aumento de problemas de saúde com o avançar da idade, assim como o uso de serviços de saúde. Dos 338 idosos participantes de um grupo da terceira idade, sendo a maioria do gênero feminino, 56,2\% relataram ter hipertensão arterial, 34,3\% dislipidemias, 32\% osteoporose, $17 \%$ câncer, $13,6 \%$ diabetes mellitus e $10 \%$ depressão.

Dentre as variáveis estudadas, somente a depressão aumentou significativamente com o aumento de risco nutricional pela classificação do NSI. Se não tratada, pode representar uma grave ameaça para a saúde geral dos idosos. O estudo transversal de Heuberger e Wong (2014) demonstrou que depressão e/ou viuvez impactaram negativamente sobre o estado nutricional de idosos residentes nos Estados Unidos, com declínio gradual no consumo energético da alimentação. Alterações psicossociais têm sido associadas com o estado socioeconômico e de saúde em idosos, contribuindo para o desinteresse das atividades diárias, entre elas o preparo das refeições e o ato de se alimentar, aumento do sedentarismo e, consequentemente, comprometimento do estado nutricional.

A maioria das idosas no presente estudo classificaram-se como fisicamente ativas e independentes, embora o nível funcional não se associou significativamente com a capacidade física das mesmas comprarem alimentos. $\mathrm{O}$ estudo de Silveira, Faro e Oliveira (2011) permite confirmar que a manutenção da capacidade funcional no envelhecimento é significativamente influenciada por aspectos físicos, mentais, psicossociais, econômicos e culturais.

Ao considerar a presença de risco nutricional através do Nutrition Screening Initiative, a maioria das idosas (51,9\%) apresentaram baixo risco, 36,5\% moderado risco e apenas $11,5 \%$ alto risco nutricional. Convém ressaltar, que as mulheres desse estudo eram em sua maioria ativas, podendo justificar o maior percentual de idosas sem risco nutricional. 
Estudos nacionais e internacionais corroboram com os resultados dessa pesquisa. No município de Passo Fundo (RS), semelhantes proporções foram encontradas, em que 55,6\% dos idosos avaliados foram classificados com baixo risco, 27,8\% com risco moderado e 16,6\% alto risco pelo NSI (STOBBE et al., 2005). Os resultados indicam que, comparando o risco nutricional moderado/ alto das idosas avaliadas de Caxias do Sul com os idosos de Passo Fundo, tem-se um risco de $48 \%$ e de $44,4 \%$, respectivamente.

Na pesquisa de Wham et al. (2014), em Nova Zelândia, constatou-se resultados divergentes, com maior prevalência de risco nutricional moderado/ alto (66,5\%) pelo NSI, dentre as 1872 idosas. Esse foi um dado preocupante, uma vez que dificilmente os indivíduos reduziriam a sua classificação de risco sem intervenção nutricional.

As pontuações atribuídas para os itens do NSI refletem na sua importância como indicador de risco nutricional. A existência de dificuldades financeiras para comprar comida no NSI recebe o maior peso (quatro pontos) considerando todas as questões do instrumento. Entretanto, nesse estudo, foi a que teve um maior índice de respostas positivas. Dentre as idosas participantes, $100 \%$ possuíam condições monetárias suficientes para comprar os alimentos. Já em relação a ingestão maior ou igual a três medicamentos diariamente sob prescrição médica e realizar as refeições sozinhas na maior parte das vezes, recebem a menor pontuação (um ponto) e foram os itens que obtiveram um elevado número de respostas positivas, sendo 61,5\% e 39,4\%, respectivamente. Resultados semelhantes foram encontrados no estudo de Azevedo e Azevedo (2014), no qual também demonstraram que 100\% dos idosos possuíam condições financeiras, $61,4 \%$ realizavam as refeições sozinhos e 29,5\% ingeriam três ou mais medicamentos diários.

O presente estudo revelou associação significativa da maioria dos itens do questionário NSI com a classificação de risco nutricional. Da mesma forma, Quigley, Hermann e Warde (2008), encontrou um aumento do percentual de respostas positivas para a maioria das questões do NSI à medida que aumentava o risco nutricional.

Wunderlich et al. (2012) enfatizaram comportamentos alimentares saudáveis e aumento significativo do consumo alimentar de frutas/verduras e produtos lácteos quando os idosos apresentaram maior renda e alto nível de escolaridade. Os resultados desse estudo apontaram através do QFA, um consumo diário de frutas, vegetais crus, leite e queijo, sendo que para o iogurte e vegetais cozidos, obteve-se uma menor frequência do consumo, de uma a três vezes por semana. As idosas que relataram baixo consumo de frutas, verduras e/ou produtos lácteos na triagem - NSI - são as que menos 
frequentemente consomem frutas pelo QFA. Através desses dados, foi possível observar a importância da adaptação do instrumento NSI com a separação dos grupos alimentares (frutas, verduras e produtos lácteos), já utilizada em alguns estudos recentes, como uma forma de melhorar a avaliação do risco nutricional (WHAM et al., 2014; WUNDERLICH; BAI; PIEMONTE, 2011). Como limitações deste estudo, entende-se que o NSI na sua forma original não separa os grupos alimentares acima citados.

A partir dos resultados desse estudo, os dados apontam que, o NSI foi aplicado à uma amostra de idosas que em sua maioria possuíam boas condições econômicas, por isso não é possível generalizar e extrapolar as informações encontradas para todos idosos, pois os dados encontrados podem não ser similares, quando aplica-se em idosos com padrões econômicos diferentes. A renda é um dos itens que mais pontua no NSI e auxilia identificando o risco nutricional. Nesse sentido, outros estudos utilizando o instrumento de avaliação de risco nutricional NSI devem ser realizados, para auxiliar no estabelecimento do diagnóstico nutricional precoce.

\section{Considerações finais}

Na presente pesquisa encontrou-se elevada prevalência de sobrepeso, obesidade e CC em risco para doenças cardiovasculares, porém um baixo risco nutricional entre as idosas pelo Nutrition Screening Initiative, sendo a depressão, quando presente, responsável pelo aumento do risco nutricional. De acordo com os resultados apresentados, torna-se necessário a realização de outros estudos, inclusive longitudinais, que estabeleçam uma avaliação multidimensional geriátrica considerando a utilização do NSI, associado à métodos de avaliação do estado nutricional para idosos. Portanto, identificar fatores potencialmente problemáticos ou de potencial risco nutricional pode permitir o direcionamento de intervenções de saúde, especialmente a nutricional, além de auxiliar agregando qualidade aos anos adicionais de vida dessa crescente parcela populacional. 


\title{
abstract
}

The assessment of nutritional status and early detection of nutritional risks that follow the female aging are considered a priority in primary health care. This study aimed to evaluate the nutritional status and nutritional risk in a sample of elderly women of the UCS Senior Program at the University of Caxias do Sul. It is a cross-sectional, descriptive and analytical study. It was used sociodemographic, anthropometric, epidemiological variables, food intake were obtained by food frequency questionnaire (FFQ) and nutritional risk screening (NSI). Among the 104 evaluated, 59.6\% had companions, $71.1 \%$ reported having completed primary education, middle or higher, $74 \%$ had income above two monthly minimum wages and $94.2 \%$ were classified as active and / or independent. In relation to BMI, 44.2\% were overweight and/or obese and only $11.5 \%$ underweight. There was high prevalence of dyslipidemia (61.5\%) and waist circumference in risk for cardiovascular disease (74\%), according to WHO (2003). Among the variables studied, only the depression increased significantly with increased nutritional risk classification by the NSI ( $p=0.002)$. The Nutrition Screening Initiative has identified 51.9\% of elderly in low-risk, 36.5\% moderate risk and $11.5 \%$ high nutritional risk. Identifying predictive factors of nutritional risk by association of indicators may allow health interventions, especially the nutritional, besides contributing to the quality of life of this growing part of the population.

keywords

Aging. Primary Health Care. Nutritional Status. Risk.

referências

\begin{abstract}
ANDRADE, Fabíola Bof de et al. Prevalence of overweight and obesity in elderly people from Vitória - ES. Ciência Saúde Coletiva, Rio de Janeiro, v. 17, n. 3, p. 749-756, mar. 2012.

ATELLA, Vicenzo; CONTI, Valentina. The effect of age and time to death on primary care costs: The Italian experience. Social Science \& Medicine, Rome, v. 114, p. 10-17, Aug. 2014.

AZEVEDO, Daniela Pousada; AZEVEDO, Cesar Henrique de. Avaliação do risco nutricional de idosos em Santos - São Paulo. In: CONGRESSO NACIONAL DE INICIAÇÃO CIENTÍFICA DA SEMESP, 14., 2014, São Paulo. Anais eletrônicos... São Paulo: Semesp, 2014. Disponível em: <http://conic-semesp.org.br/anais/files/2014/trabalho-1000016719. pdf $>$. Acesso em: 13 jun. 2015.
\end{abstract}


BRASIL. Ministério da Saúde. Orientações para a coleta e análise de dados antropométricos em serviços de saúde: Norma Técnica do Sistema de Vigilância Alimentar e Nutricional - SISVAN. Brasília, DF: Ministério da Saúde, 2011. (Série G. Estatística e Informação em Saúde).

BRETON, Émilie et al. Gender-specific associations between functional autonomy and physical capacities in independent older adults: Results from the NuAge study. Archives of Gerontology and Geriatrics, Toronto, v. 58, n. 1, p. 56-62, Jan./Feb. 2014.

DEAN, Moira et al. Factors influencing eating a varied diet in old age. Public Health Nutrition, Toronto, v. 12, n. 12, p. 2421-2427, Apr. 2009.

DIGIACOMO, Michelle et al. The business of death: a qualitative study of financial concerns of widowed older women. BMC Women's Health, London, v. 15, n. 1, Apr. 2015.

GONÇALVES, Déborah Franco et al. Nutritional status and epidemiological profile of elderly people. Archives of Gerontology and Geriatrics, Rio de Janeiro, v. 55, n. 1, p. 1-4, Jul./Aug. 2012.

HEUBERGER, Roschelle; WONG, Helen. The association between depression and widowhood nutritional status in older adults. Geriatric Nursing, Grand Rapids, v. 35, n. 6, p. 428-433, Nov./Dec. 2014.

$\mathrm{KIM}, \mathrm{Hae}$ Jin et al. Relation between nutritional risk and metabolic syndrome in the elderly. Archives of Gerontology and Geriatrics, Seul, v. 52, n. 1, p. 19-22, Jan./Feb. 2011.

KUERBIS, Alexis et al. Substance Abuse Among Older Adult. Clinics in Geriatric Medicine, Rockville, v. 30, n. 3, p. 629-654, Aug. 2014.

MARENGONI, A. et al. Understanding adverse drug reactions in older adults through drug-drug interactions. European Journal of Internal Medicine, Milan, v. 25, n. 9, p. 843-846, Nov. 2014.

MATSUDO, Sandra Mahecha. Avaliação do idoso: Física \& Funcional. 2. ed. Londrina: Midiograf, 2000, p. 125.

ORGANIZAÇÃO PAN-AMERICANA DE LA SALUD (OPAS). Encuesta Multicêntrica: Salud Bienestar y Envejecimento (SABE) em América Latina y el Caribe. In: INFORME PRELIMINARY DA REUNIÓN DEL COMITÉ ASESOR DE INVESTIGACIONES EM SALUD, 36., 2001, Washington, D.C. Anais eletrônicos... Washington, D.C.: Organización Panamericana de la Salud, 2001. Disponível em: <http://envejecimiento.csic.es/documentos/ documentos/paho-salud-01.pdf>. Acesso em: 13 jun. 2015.

PEREIRA, Érico Felden et al. Relação entre diferentes indicadores antropométricos e a percepção da imagem corporal em idosas ativas. Revista de Psiquiatria Clínica, São Paulo, v. 36, n. 2, p. 54-59, 2009.

POSNER, Barbara Millen et al. Nutrition and health risks in the elderly: the nutrition screening initiative. American Journal Public Health, Washington, v. 83, n. 7, p. 972-978, July 1993.

QUIGLEY, Kimberly K.; HERMANN, Janice R.; WARDE, Willian D. Nutritional risk among Oklahoma congregate meal participants. Journal of Nutrition Education and Behavior, New York, v. 40, n. 2, p. 89-93, Mar./Apr. 2008.

SANTOS, Adriane Heldt dos et al. Triagem nutricional: identificação de alterações nutricionais na atenção à saúde do idoso. Revista Brasileira de Ciências do Envelhecimento Humano, Passo Fundo, v. 10, n. 2, p. 170-180, maio/ago. 2013.

SHERINA, Mohd Sidik et al. The Association of nutritional risk physical and mental health problems among elderly in a semi-urban area of Mukim Kajang, Selangor, Malaysia. Malaysian Journal of Nutrition, Seri Kembangan, v. 10, n. 2, p. 149-158, 2004.

SILVEIRA, Solange Convento; FARO, Ana Cristina Mancussi; OLIVEIRA, Claudia Lysia Araújo. Atividade física, manutenção da capacidade funcional e da autonomia em idosos: revisão de literatura e interfaces do cuidado. Estudos Interdisciplinares sobre o Envelhecimento, Porto Alegre, v. 16, n. 1, p. 23-39, jun. 2011. 
STOBBE, Júlio César et al. Projeto Passo Fundo-RS: indicadores de saúde de participantes de um grupo de terceira idade. Revista Brasileira de Ciências do Envelhecimento Humano, Passo Fundo, v. 2, n. 1, p. 89-101, jan./jun. 2005

TANJANI, Parisa Taheri et al. The health status of the elderly population of Iran in 2012. Archives of Gerontology and Geriatrics, Radarweg, v. 60, n. 2, p. 281-287, Mar./Apr. 2015.

WHAM, C. A et al. The BRIGHT trial: what are the factors associated with nutrition risk? The Journal of the Nutrition, Health \& Aging, Berlin, v. 18, n. 7, p. 692-697, Sep. 2014.

WHO/FAO Expert Consulation. Diet, Nutrition and prevention of chronic diseases. Rome: World Health Organization (Technical Report Series, n. 916), 2003.

WUNDERLICH, Shala; BAI, Y; PIEMONTE, J. Nutrition risk factors among home delivered and congregate meal participants: need for enhamcement of nutrition education and counseling among home delivered meal participants. The Journal of Nutrition, Health \& Aging, Montclair, v. 15, n. 9, p. 768-773, Nov. 2011

WUNDERLICH, Shahla et al. Eating Behaviors of Older Adults Participating in Government-Sponsored Programs with Different Demographic Backgrounds. Global Journal of Health Science, Toronto, v. 4, n. 6, p. 204-215, Mar. 2012.

YAP, K. B.; NITI, M. Nutrition screening among community-dwelling older adults in Singapore. Singapore Medical Journal, Bukit Merah, v. 48, n. 10, p. 911-916, Oct. 2007.

ZOGHBI, Mohamad El et al. Association between cognitive function and nutritional status in elderly: A cross-section study in three institutions of Beirut-Lebanon. Geriatric Mental Health Care, Amsterdam, v. 1, n. 4, p. 73-81, Dec. 2013.

Data de Submissão: 10/11/2015

Data de Aprovação: 24/03/2018 